\title{
Invasão do Reto por Carcinoma Prostático Avançado com Disseminação Linfática Simulando Câncer Retal - Relato de Caso
}

\author{
Invasive Prostate Carcinoma to the Rectum with Lymphatic \\ Dissemination Simulating a Rectal Cancer - Case Report
}

\author{
LEONARDO MACIELDAFONSECA ${ }^{1} ; \dagger$ ANAMARGARIDAMIGUELF. NOGUEIRA ${ }^{2}$; BERNARDO HANAN ${ }^{3}$; \\ MAGDAMARIAPROFETADALUZ ${ }^{4}$; RODRIGO GOMES DASILVA ${ }^{5}$; ANTÔNIO LACERDA-FILHO ${ }^{6}$ \\ ${ }^{1}$ Médico Coloproctologista, pós graduando em cirurgia da Faculdade de Medicina da UFMG; ${ }^{2}$ Professora doutora \\ do Departamento de Anatomia Patológica da Faculdade de Medicina da Universidade Federal de Minas Gerais \\ († in memorian); ${ }^{3}$ Médico coloproctologista $;{ }^{4}$ Titular da Sociedade Brasileira de Coloproctologia; ${ }^{5}$ Professor \\ Doutor do Departamento de Cirurgia da Faculdade de Medicina da UFMG, Coordenador do Grupo de \\ Coloproctologia e Intestino Delgado do Instituto Alfa de Gastroenterologia do Hospital das Clínicas da UFMG; ${ }^{6}$ \\ Professor Doutor do Departamento de Cirurgia da Faculdade de Medicina da UFMG, Titular da Sociedade \\ Brasileira de Coloproctologia.
}

FONSECA LM; NOGUEIRA AMMF; HANAN B; LUZ MMP; SILVA RG; LACERDA-FILHO A. Invasão do Reto por Carcinoma Prostático Avançado com Disseminação Linfática Simulando Câncer Retal - Relato de Caso. Rev bras Coloproct, 2010;30(1): 074-078.

RESUMO: O carcinoma da próstata é uma doença freqüente em idosos e em casos avançados pode invadir o reto, simulando um carcinoma primário deste órgão. Nestas situações, a maioria dos pacientes apresenta sintomas retais exuberantes e sintomas urinários leves ou ausentes. É relatado um caso de um paciente com diagnóstico de tumor de próstata localmente agressivo, concomitante a um tumor viloso retal, que foi diagnosticado e tratado erroneamente como um tumor primário do reto. Tal lesão, curiosamente, apresentava comportamento biológico compatível com câncer retal, inclusive com disseminação linfática típica desta doença. A incidência relatada de invasão do reto por tumores de próstata avançados varia entre 1 e $11 \%$ em diferentes séries. $O$ aspecto do tumor de próstata com envolvimento retal traz dificuldades em diferenciá-lo de um tumor primário. Exames radiológicos e ou endoscópicos podem não esclarecer o diagnóstico, enquanto o exame histopatológico em ambos os tumores costuma revelar adenocarcinoma pouco diferenciado. A diferenciação diagnóstica entre estes dois tumores é essencial, uma vez que o tratamento é absolutamente diferente para as duas doenças. A alta incidência do carcinoma prostático o torna importante para que todos os médicos estejam atentos à possibilidade deste tumor invadir o reto e simular um tumor primário deste órgão.

Descritores: Neoplasia Prostática, Neoplasia retal, Cirurgia Colorretal, Próstata, Reto.

\section{INTRODUÇÃO}

O carcinoma de próstata é uma doença freqüente em idosos e em casos avançados pode invadir o reto e simular um tumor primário deste órgão. A maioria dos pacientes apresenta sintomatologia retal exuberante, como diarréia, sangramento, constipação ou tenesmo, enquanto os sintomas urinários são mínimos ou ausentes. Quando as manifestações clínicas são predominantemente intestinais, geralmente o carcinoma de próstata apresenta-se de forma avançada. A distinção entre estes dois tumores é de fundamental importância uma vez que o tratamento é absolutamente distinto. Um alto índice de suspeição é necessário para que o diagnóstico seja obtido.

É relatado a seguir um caso de um paciente com diagnóstico de tumor de próstata localmente agressivo, invasor do reto, concomitante a um tumor viloso

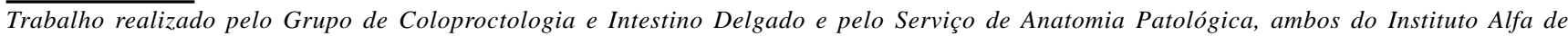
Gastroenterologia do Hospital das Clínicas da Universidade Federal de Minas Gerais, Belo Horizonte/MG - Brasil. 
Rev bras Coloproct

Janeiro/Março, 2010 deste órgão, que foi diagnosticado e tratado erroneamente como um tumor primário retal. As vias de disseminação seguidas por este tumor foram típicas de um câncer retal.

\section{RELATO DE CASO}

Trata-se de paciente de 73 anos encaminhado para avaliação coloproctológica com história de diarréia muco-sanguinolenta, associada a tenesmo há cerca de quatro anos. Não havia relato de perda de peso, dor abdominal, febre ou alteração da função urinária. Apresentava história médica pregressa irrelevante e exame físico geral sem anormalidades. O toque retal evidenciou volumosa lesão retal, anular em parede retal anterior que se iniciava a $1,5 \mathrm{~cm}$ da margem anal. À anuscopia foi observada uma massa retal de aspecto viloso, que à biópsia revelou adenoma túbulo-viloso com displasia de baixo e de alto grau. Realizada colonoscopia que evidenciou lesão retal de espraiamento lateral, que se iniciava logo acima da linha pectínea, com superfície avermelhada, friável e que cobria cerca de $2 / 3$ da circunferência do reto, estendendo-se até $3 \mathrm{~cm}$ cranialmente. O restante do cólon encontrava-se sem alterações. Foi realizada também ultra-sonografia endoscópica que revelou lesão hipoeicoíca infiltrativa da mucosa com suspeita de invasão da submucosa. $\mathrm{O}$ paciente foi submetido à ressecção endoscópica da lesão pela técnica de peacemeal em três sessões, sendo o tratamento complementado com fotocoagulação por plasma de argônio. O exame anátomo-patológico confirmou tratar-se de adenoma túbulo-viloso com displasia de baixo e de alto grau.

Após a ressecção endoscópica, o paciente apresentou estenose anal progressiva e com má resposta à dilatação digital e com velas de Hegar. Foi readmitido cerca de cinco meses após o procedimento com queixa de dor abdominal, tenesmo, sangramento anal e incontinência fecal. Queixava-se ainda de dorsalgia e disúria. O exame retal revelou lesão estenosante, circunferencial, irregular e endurecida localizada no reto distal que impedia a passagem do dedo. O paciente foi submetido à ressecção parcial do tecido fibrótico e o exame histopatológico mostrou tratar-se de um carcinoma pouco diferenciado. Tomografia computadorizada do abdome e pelve demonstrou pequeno nódulo em segmento hepático III/IV compatível com metástase e um espessamento da parede retal, com ausência de plano de clivagem em relação à prós- tata e à bexiga (Figuras 1 e 2). A cintilografia óssea foi negativa para lesões osteolíticas. A dosagem sérica do antígeno cárcino-embrionário (CEA) foi de 3,0 ng/ml.

Diante dos achados, firmou-se diagnóstico de carcinoma retal avançado, presumindo-se que o tratamento endoscópico do tumor viloso tinha sido inadequado. O paciente foi levado à cirurgia, na qual identificou-se suposto tumor retal com invasão grosseira da bexiga, próstata e vesículas seminais (figura 3). Procedeu-se então à exenteração pélvica com amputação abdômino-perineal do reto e desvio urinário através da construção de ureterocolostomia dupla em alça cega ("colostomia úmida").

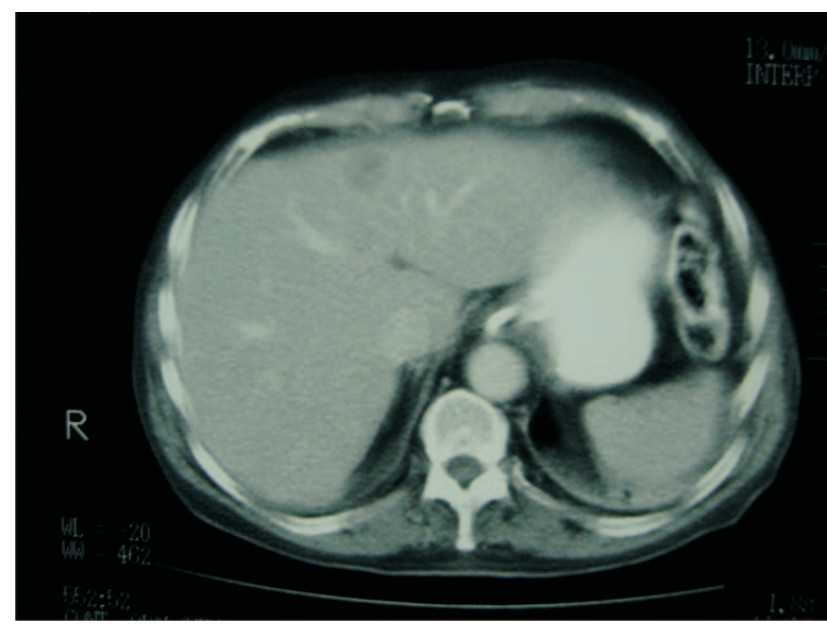

Figura 1- Tomografia de abdome mostrando nódulo em segmentos hepático III/IV compatível com metástase.

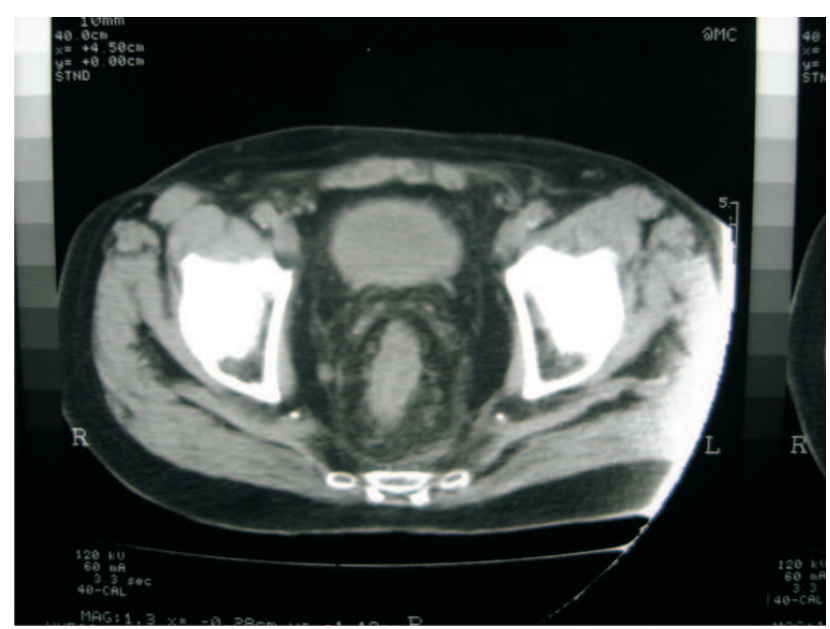

Figura 2- Tomografia computadorizada de pelve mostrando espessamento da parede retal. 
O exame anátomo-patológico da peça cirúrgica revelou tratar-se de um adenocarcinoma prostático infiltrativo, pouco diferenciado, Gleason $9(5+4)$ (Figura 4), com invasão maciça do reto, bexiga e vesículas seminais, com metástase em 42 de 52 linfonodos perirretais e mesentéricos dissecados. A origem prostática foi confirmada com estudo imunohistoquímico. Não foi realizada biópsia hepática devido ao pequeno tamanho do nódulo e também porque se assumiu que a metástase era de origem retal. $\mathrm{O}$ paciente apresentou recuperação pós-operatória lenta, contudo sem complicações maiores. As avaliações urológica e oncológica não indicaram tratamento complementar, devido ao caráter agressivo do tumor prostático.

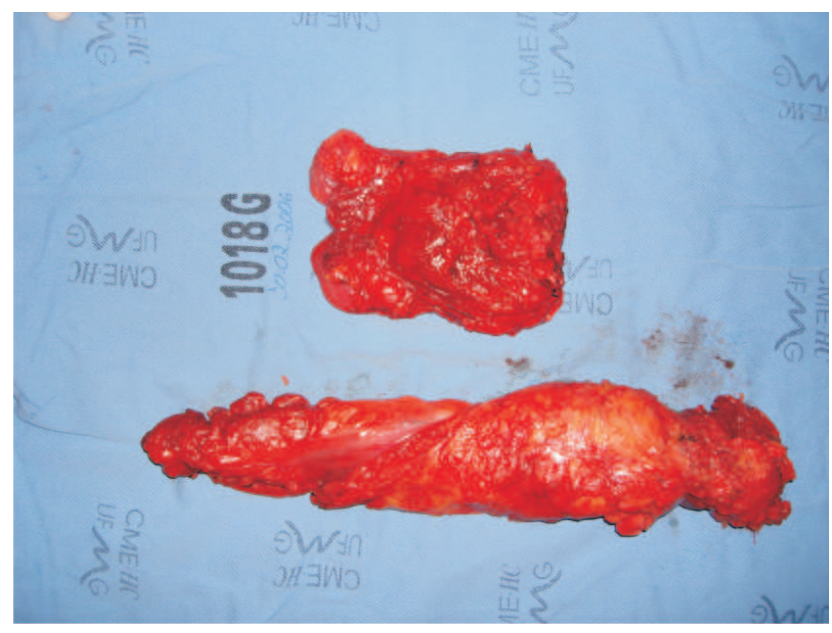

Figura 3-Peça cirúrgica - .Bexiga, próstata e vesícula seminal em bloco acima e anus, reto e segmento distal do sigmóide abaixo.

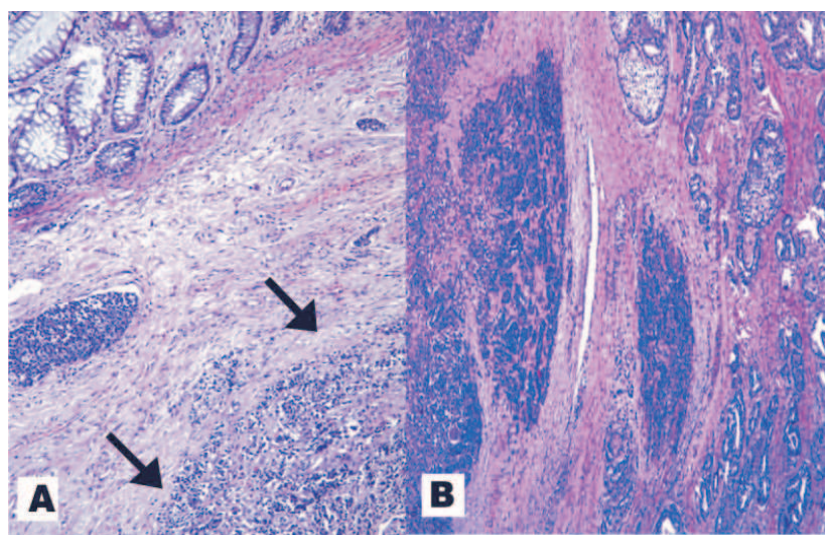

Figura 4- A) Infiltração da submucosa retal pelo tumor (setas). A mucosa retal esta bem preservada. B) Histologia do tumor, constituído por massa sólida de células indiferenciadas (à esquerda). Em menor proporção o tumor apresenta glândulas atípicas justapostas, com ausência de estromas entre elas (à direita).
O paciente recebeu alta hospitalar 29 dias após a cirurgia e foi readmitido dez dias após, devido a quadro de pneumonia de aspiração que evoluiu para insuficiência respiratória. Sua condição deteriorou-se progressivamente, tendo evoluído para o óbito 13 dias depois.

\section{DISCUSSÃO}

A incidência relatada de invasão retal por carcinoma de próstata varia entre 1 e $11 \%$ em diferentes séries $^{(1,2,3,4,5,6,7)}$. Esta baixa incidência, apesar da proximidade anatômica destes dois órgãos, deve-se à barreira de proteção exercida pela fáscia de Denonvilliers, que é composta por duas camadas e, na maioria dos casos, impede o crescimento posterior dos tumores prostáticos. Contudo, tumores agressivos podem penetrar esta fáscia e invadir o reto, levando ao aparecimento de sintomas retais. A invasão retal por adenocarcinoma de próstata é um marcador de doença avançada e poucos pacientes sobrevivem mais de 30 meses $^{(3)}$.

As formas de invasão do retossigmóide por tumores de próstata foram definidas em três tipos, por Lazarus em $1935^{(8)}$ : tipo I - massa retal anterior; tipo II - estenose retal anular e tipo III - massa em parede retal anterior ulcerada. Em 1957, Winter ${ }^{(7)}$ acrescentou mais dois tipos à classificação de Lazarus, tipo IV, para denominar metástase isolada para o retossigmóide e tipo $\mathrm{V}$ para carcinomas de próstata e de reto associados. A distribuição entre estes grupos é de $25 \%, 40 \%$, $21 \%, 9 \%$ e $3 \%$ respectivamente ${ }^{(7)}$. O caso relatado pode ser enquadrado tanto no tipo II, quanto no tipo V, uma vez que a presença de um grande adenoma túbuloviloso no reto foi um fator de confusão, que acarretou diagnóstico e tratamento inadequados.

$\mathrm{O}$ aspecto deste tumor prostático com envolvimento agressivo do reto causou dificuldades em sua diferenciação de um tumor retal primário. Além disso, as manifestações retais suprimiram os sintomas do trato geniturinário, o que representou mais um fator de confusão. Esta observação está de acordo com o trabalho de Papa et al. ${ }^{(9)}$ que estudaram retrospectivamente dez pacientes com tumores de próstata invadindo o reto. Nesta série, todos os pacientes apresentaram sintomas abdominais e retais, enquanto apenas quatro tinham sintomas urinários, como disúria, polaciúria ou hematúria.

No presente caso, como a biópsia realizada na área estenótica do reto revelou a presença de carcinoma anaplásico, a impressão foi da possibilidade de 
Rev bras Coloproct

Janeiro/Março, 2010 recorrência ou de resíduo local do tumor retal, previamente tratado, embora a histopatologia não confirmasse o caráter invasivo da lesão ressecada. . O diagnóstico histopatológico exato, especialmente quando o tumor é muito anaplásico, é bastante difícil. Tanto tumores prostáticos invasivos do reto, quanto tumores retais primários avançados, usualmente são vistos como adenocarcinomas pouco diferenciados e, freqüentemente, apenas o estudo imunohistoquímico para antígeno prostático específico (PSA) ou fosfatase ácida prostática podem diferenciar estes dois tumores ${ }^{(10)}$. Desta forma, em casos sem definição diagnóstica, o estudo imunohistoquímico do espécime de biópsia deve ser realizado de forma sistemática.

Também a forma de disseminação linfática neste caso representa um interessante comportamento biológico do tumor prostático, que além de envolver o reto, disseminou-se através da rede de drenagem linfática deste órgão, com metástase em 42 de 52 linfonodos perirretais e mesentéricos dissecados.

A diferenciação entre tumor primário retal e tumor prostático com envolvimento retal é fundamental, uma vez que o tratamento para as duas doenças difere completamente. Enquanto o carcinoma retal requer abordagem cirúrgica radical, a melhor conduta pra o tumor de próstata avançado resume-se apenas a orquiectomia e/ou hormonioterapia. Isto porque, o envolvimento retal por um carcinoma da próstata representa doença incurável, e é contra-indicado o tratamento cirúrgico radical ${ }^{(5)}$. Infelizmente, devido ao erro diagnóstico, o presente caso foi abordado como se o tumor fosse de origem retal primária, conduta esta absolutamente inadequada para tratar o verdadeiro tumor, que era de origem prostática.

Este problema é enfatizado por vários outros autores que estudaram casos de pacientes portado- res de tumores de próstata com invasão retal submetidos à ressecção abdominoperineal do reto devido a diagnóstico incorreto ${ }^{(7,11,12,13)}$. Bowrey et al.$^{(3)}$ relataram uma série de 6 pacientes com tumores de próstata que invadiam o reto. Em um caso houve confusão diagnóstica e o paciente foi submetido a uma ressecção colorretal inadequada supostamente para tratar um tumor retal primário, e não um tumor de próstata invasor do reto. De acordo com estes autores, em uma revisão da literatura com um total de 155 pacientes portadores de tumores de próstata invadindo o reto, 41 destes necessitaram tratamento cirúrgico. Quatro pacientes foram submetidos à ressecção abdômino-perineal do reto e um a um ressecção anterior devido a diagnóstico pré-operatório incorreto de carcinoma primário do reto.

\section{CONCLUSÃO}

Os sinais e sintomas clínicos decorrentes da invasão retal por um tumor prostático não são totalmente claros em relação a real origem deste tumor. A apresentação clínica mais freqüente é caracterizada por quadro constituído por sintomas usualmente relacionados ao câncer de reto. Exames radiológicos e/ou endoscópicos são incapazes de fornecer um diagnóstico definitivo. A biópsia retal geralmente demonstra adenocarcinoma pouco diferenciado e a conclusão é obtida com emprego de estudos específicos, utilizando fosfatase ácida ou PSA. Assim, em casos com alto índice de suspeição, a diferenciação se faz necessária, de forma que uma ressecção colorretal inapropriada não seja realizada. A alta incidência dos tumores de próstata torna-o importante para que todos os médicos estejam atentos à possibilidade destes tumores invadir e simular um tumor primário do reto.

\footnotetext{
ABSTRACT: Prostate carcinoma is a frequent disease in the elderly and in advanced cases it can cause rectal invasion mimicking a primary rectal carcinoma. Most of patients present with significant rectal symptoms and mild to absent urinary tract symptoms. We report a case of a patient with a very aggressive locally invasive prostate carcinoma with a concomitant rectal villous tumor which was misdiagnosed and inadequately treated as a rectal cancer. The reported incidence of rectal invasion in advanced prostate cancer has varied between 1 to 11 per cent in different series. The appearance of the prostate tumor with involvement of the rectum causes difficulties in differentiating it from primary rectal carcinoma. Neither radiological nor endoscopic examination of the rectum or lower urinary tract provides a definitive diagnosis. Histopathology in both primary prostate carcinoma invasive to the rectum and primary rectal carcinoma usually is poorly differentiated adenocarcinoma.The differentiation of rectal involvement from prostatic carcinoma is essential, since therapy is quite different for the two diseases. The high incidence of prostatic carcinoma makes it important for all physicians are aware of frequency in which it involves the rectum and mimic a primary rectal neoplasm.
}

Key words: Prostatic Neoplasms, Rectal Neoplasms, Colorectal Surgery, Prostate, Rectum. 


\section{REFERÊNCIAS}

1. Oslen BS, Carlisle RW. Adenocarcinoma of the prostate simulating primary rectal malignancy. Cancer 1970;25:21922.

2. Lasser A. Adenocarcinoma of the prostate involving the rectum Dis Colon Rectum 1978;21:23-5.

3. Bowrey DJ, Otter MI, Billings PJ. Rectal infiltration by prostatic adenocarcinoma: report on six patients and review of the literature. Ann R Coll Surg Engl 2003;85:382-5.

4. Surya BV, Provet JA. Mabifestattions of advanced prostate cancer: prognosis and treatment. J urol 1989;142:921-8.

5. Becker JA. Prostatic carcinoma involving the rectum and sigmoid colon. Roent-Genol Rad Therapy \& nuclear med 1965;94:421-8.

6. Roberts RA, Norman RW, Mack FG. Rectal obstruction by prostatic carcinoma: diagnosis by computerized tomography scan. J Urol 1986;135:137-9.

7. Winter CC. The problem of rectal involvement by prostatic cancer. Surg Gynec \& Obst 1957;105:136-40.

8. Lazarus JA. Complete rectal occlusion necessitating colostomy due to carcinoma of the prostate. Am J Surg 1935;30:502-5.
9. Papa MZ, Koller M, Klein E, Bersuck D, Sarely M, Arie GB. Short Note: Prostatic Cancer Presenting as a Rectal Mass: A Surgical Pitfall. Br J Surgery 1997;84:69-70.

10. Siegel AL, Tomaszewski JE, Wein AJ, Hanno PM. Invasive carcinoma of prostate presenting as rectal carcinoma. Urology 1986;27:162-4.

11. Fry DE, Amin M, Harbrecht PJ. Rectal obstruction secondary to carcinoma of the prostate. Ann Surg 1979;189:488-92

12. Mir M, Dikranian H, Cogbill CL. Carcinoma of the prostate as obstructive carcinoma of the rectum. Am Surg 1973;39:5826.

13. Reayer ER, Utley WL. Rectal manifestations of the malignant prostate. Aust N Z J Surg 1955;24:272-8.

\section{Endereço para correspondência:}

PROF. DR. ANTÔNIO LACERDA-FILHO

Instituto Alfa de Gastroenterologia

Hospital das Clínicas da UFMG - $2^{\circ}$ andar

Av. Alfredo Balena, 110

Belo Horizonte/MG

BRASIL CEP 30130-100

Tel: 55-31-32489403

Fax: 55-31-32489251

E-mail: alacerda@ufmg.br 